Biological therapies of the inner ear; what otologists need to consider.

Anne GM Schilder ${ }^{1}$, Helen Blackshaw ${ }^{1}$, Thomas Lenarz $^{2.3}$, Lawrence Lustig ${ }^{4}$, Hinrich Staecker ${ }^{5}$, Athanasia Warnecke $e^{2,3}$

'evidENT, Ear Institute, University College London, UK 2Department of Otolaryngology Head and Neck Surgery, Hannover Medical School, Hannover, Germany

${ }^{3}$ Cluster of Excellence "Hearing for all", German Research Foundation

${ }^{4}$ Department of Otolaryngology Head and Neck Surgery, Columbia University, New York, USA ${ }^{5}$ Department of Otolaryngology Head and Neck Surgery, University of Kansas Medical Center, Kansas City, USA

Keywords: Biological Therapies, Inner Ear, Regeneration, Clinical Trials

Over the past 50 years, our understanding of the molecular and cellular basis of hearing has grown immensely. While for decades it has been a dream to 'regrow' portions of the inner ear to treat hearing loss, biological therapies for the protection and regeneration of the inner ear are now on the horizon; with an active pipeline of drug, gene and cell therapies now moving towards clinical translation. This poses new challenges to clinicians and researchers in the hearing field. In this commentary, we share some of our experiences in developing and conducting early phase trials of novel hearing therapies and suggest a collaborative approach to ensure optimal enhanced benefit to our patients.

Hearing loss and the regulation of hearing loss therapies have become a public priority for governments worldwide. Recent data shows that approximately half a billion people have disabling hearing loss, roughly corresponding to $6-8 \%$ of the world's population [1] with an estimated annual economic burden of 750 billion US Dollars. This impact 
is set to increase as the world's population ages and consequently the number of people with age-related hearing loss grows [2,3]. Importantly, adult onset hearing loss has recently been identified as a key risk factor for dementia [4].

The life sciences industry too has realised that hearing health care is an area of unmet need and great potential profit. This is evidenced by the fact that financial investments in new hearing loss therapies have risen sharply with more than 30 biotechnology start-ups generated in the hearing field over the past several years [5]. With these investments, new drugs, gene and cellular therapies are being developed to prevent, alleviate or restore hearing loss and tinnitus. Translating these discoveries to the clinical domain creates new opportunities for otologists and neurotologists, since expertise in hearing health care will be needed for both the development of these new interventions as well as approaches to the delivery of these therapies to the inner ear.

Particular challenges faced at the early stages of translation can be roughly divided into the areas of regulatory and financial hurdles, limitations in our current understanding of hearing loss mechanisms, and the need to develop internationally accepted standards for efficacy and outcomes analyses.

\section{Regulatory hurdles}

Moving a new therapy from the bench to the bedside is a lengthy and resource intensive process, where input from experts in drug development, or those who have successfully guided gene or cellular therapies in other conditions through the approval process, is essential. With novel hearing therapies also being a new area for contract research organisations (CROs) and regulatory bodies, direct clinical guidance by those involved in these trials will be vital throughout this process.

To achieve US Food and Drug Administration (FDA) approval for novel compounds or approaches, sufficient basic and preclinical research needs to be conducted. Data on dose-response, safety and efficacy needs to be presented in a standardized and reproducible fashion to the authorities. Only if this suffices, can next steps towards clinical trials be considered.

Conducting first-in-human studies is bound by a 
framework of national and international regulatory and governance arrangements aimed at securing patient safety. While commercial or university clinical research facilities (CRFs) are designed to operate according to these standards, they may not have the capacity to host the specific hearing equipment required to test safety in terms of hearing and balance in our trials (e.g., soundproof rooms with audiometers, rotatory chairs). The alternative, conducting these studies within Otolaryngology departments safely and according to these standards, requires regulatory expertise and resources that may be out of reach for a majority of centres. Thus it is likely that partnerships with the private sector will be critical to allow these studies to move forward.

\section{Financial hurdles}

The cost of bringing these new hearing therapies into the clinical domain will be enormous. A recent study from JAMA Internal Medicine found that the average cost of bringing a new cancer drug to market has been estimated at $\$ 650$ million, with prior estimates ranging as high as $\$ 2.7$ billion [6]. Thus, hundreds of millions of dollars will need to be raised to fund these preclinical and clinical studies, through grants from public and private funding agencies, big pharma and/or private investors.

\section{Limitations of our understanding of hearing loss pathophysiology}

Beyond the costs, selection of the most appropriate patient populations for these trials is complex. Traditionally, phase 1 drug trials recruit healthy volunteers to test the safety of a new drug. With many of the regenerative approaches requiring surgical access to the inner ear, it is more appropriate to include patients with hearing loss. With hearing loss not beeing 'a disease', but a variety of symptoms with many underlying pathologies, developing targeted therapies and selecting the matching patient groups will be one of the most difficult tasks facing our field. Selection of the appropriate populations of patients with hearing loss for these targeted therapies will require a better categorization of hearing loss based on pathophysiology. It is becoming increasingly clear that currently employed auditory measures (e.g., audiogram, ECOG) are 
insufficient to stratify patients based on hearing loss pathophysiology. Instead, this will need to be accomplished through multidimensional analysis of patient data, including phenotyping and genotyping of our patients with hearing loss, creating international data repositories of systematically collected clinical hearing data and combining these data with biorepositories of blood samples and tissue specimens for future genomic, proteomic and metabolomic analysis. These varied data sets can in turn be correlated with treatment outcomes. Only through these multidimensional measures will our understanding of the varied genetic and metabolic mechanisms that lead to hearing loss improve, and allow successful implementation of targeted molecular therapies. Networked biobanks allow not only for the sustained development of individualized and cuttingedge diagnostic methods and therapies, but also for the national and international exchange of biomaterials. Unifying these biobanks, for example by introducing them into national and international cohort programmes, could enable the collection of vast numbers of samples. Ethical and legal standards as well as standards for the quality of the biomaterials and for the exchange would need to be established among the participating centres.

Development of internationally accepted standards for efficacy and outcomes analyses

Lastly, there should be international agreement on the adequacy of measures of safety and efficacy. In drug development there is a wealth of experience in measuring systemic safety; this can be applied to the systemic hearing drugs that are being repurposed from other disease areas. For therapies topically administered to the inner ear, such as biohybrid devices for enhancement of the therapy with auditory implants (e.g., cochlear implants combined with steroids or antiapoptotic inhibitors to prevent hearing loss or growth factors to improve neuronal survival), we need better and agreed measures of local safety that include hearing and balance. Current hearing tests have been in use for decades and have limited value in predicting outcome (i.e., which patients might have the best capacity to regenerate their inner ear cells) and may not be able to pick up the subtle changes in hearing that patients may experience in challenging listening environments. Accurate electrophysiological tests of hair cell and spiral 
ganglion neuron function and neurophysiological test of auditory responses will be absolutely needed for these interventions.

Regeneration of the peripheral auditory system is a nearing reality, driven by growing concerns about the global burden of hearing loss, its impact on people and the economy, as well as by discoveries and investments in the development of novel approaches to treating hearing loss. These new therapies have the potential to transform the way hearing health care is delivered.

To achieve this reality will require a collaborative, international approach to move this field forward and establish expert communities to share experience and agree on standards for these trials. We support initiatives like the Pharmaceutical Interventions for Hearing Loss (PIHL) [7] and towards this goal, we are proposing the development of a new group; the International Society for Biological Therapies, to address these challenges head on. A first official meeting of the Society will be held in February 2018 in conjunction with the mid-winter meeting of the Association for Research in Otolaryngology (ARO) in San Diego, US. The goal of this meeting will be to bridge translational and clinical research approaches, discuss the hurdles of implementing clinical trials in this nascent field and to deliver an international consensus for biological therapies of the inner ear.

\section{References}

[1] B. Wilson, D. Tucci, M. Merson, and GM

O’Donoghue, Lancet., pii: S0140 (2017).

[2] WHO, (2015).

[3] WHO, (2017).

[4] G. Livingston, A. Sommerlad, V. Orgeta, S. Costafreda, J. Huntley, D. Ames, C. Ballard, S. Banerjee, A. Burns, J. Cohen-Mansfield, C. Cooper, N. Fox, L. Gitlin, R. Howard, H. Kales, E. Larson, K. Ritchie, K. Rockwood, E. Sampson, et al., Lancet., doi: 10.10 (2017).

[5] https://www.biocentury.com/biocentury/productdevelopment/2017-06-02/how-hearing-loss-becameinvestable-space, (2017).

[6] https://hearing.health.mil/Research/PIHL-WorkingGroup, (2017).

[7] V. Prasad and S. Mailankody, JAMA Intern. Med., 177 
(2017) 1569. 